\title{
Die Bestimmung des Aminostickstoffs in einlgen Polypeptiden nach der Methode von van Slyke.
}

\author{
Von \\ Emil Abderhalden und Donald D. van Slyke, \\ from the Laboratories of the Rockefeller Institute for Medical Research, \\ New York.
}

(Aus dem physiologischen Institut der tierärztlichen Hochschule, Berlin.)

(Der Redaktion zugegangen am 17. August 1911.)

Der eine (S.) von uns hat kürzlich eine Methode mitgeteilt, welche gestattet, den aliphatischen Aminostickstoff von Aminosäuren und Polypeptiden auf Grund der Reaktion: $\mathrm{RNH}_{2}+\mathrm{HNO}_{2}=\mathrm{ROH}+\mathrm{H}_{2} \mathrm{O}+\mathrm{N}_{2}$, in kurzer Zeit exakt zu bestimmen. Kleine Mengen genügen zur Analyse. Es schien uns von Interesse, an einem größeren Materiale bekannter Polypeptide die allgemeine Verwendbarkeit der Methode zu prüfen. Die folgende Tabelle gibt einen Überblick über die gefundenen Werte. Sie zeigen, daß die gefundenen Stickstoffzahlen sehr gut mit den berechneten übereinstimmen. Eine Ausnahme machen nur diejenigen Polypeptide, in denen Glykokoll die Aminogruppe trägt. Hier fanden wir stets etwas zuviel Stickstoff. Die Differenz ist jedoch nicht sehr groß. Vor allen Dingen nimmt sie mit der Länge der Kette der Polypeptide nicht $z u$, sondern eher ab. Es wird vielleicht möglich sein, bei noch größerer Erfahrung an einem großen Materiale für Glycyl-polypeptide einen Korrektionsfaktor anzugeben. (Vgl. hierzu Tab. 2.) Unsere Resultate zeigen jeden- 
falls, daß der von van Slyke ${ }^{1}$ ) ausgearbeiteten Methode eine große Bedeutung zukommt. Sie wird bei der Verfolgung von Fermentspaltungen und speziell auch bei der Untersuchung von Abbauprodukten, die bei der partiellen Hydrolyse der Proteine entstehen, große Dienste leisten. In letzterer Hinsicht sei darauf hingewiesen, daß das von dem einen von uns (A.) aus Seide isolierte d-Alanyl-glycin sehr scharfe Werte lieferte und ferner auch das aus Edestin gewonnene, aus Leucin, Tryptophan und Glutaminsäure bestehende Tripeptid gleichfalls ein ausgezeichnetes Resultat ergab. In der früheren Mitteilung ${ }^{2}$ ) ist die Frage offen gelassen worden, ob das isolierte Tripeptid ganz rein war. Auf Grund dieser Analyse dürfen wir jetzt annehmen, daß ein vollständig reines Tripeptid, bestehend aus l-Leucin, d-Glutaminsäure und l-Tryptophan, vorlag. ${ }^{3}$ ) Nur über die Reihenfolge, in der die Aminosäuren sich folgen, läßt sich noch nichts Bestimmtes aussagen. Es ist dies das zweite Tripeptid, das aus Eiweißabbauprodukten gewonnen werden konnte. ${ }^{4}$ )

Die einzelnen Bestimmungen wurden genau nach der angegebenen (l. c.) Vorschrift ausgeführt. Die Dauer der Reaktion betrug gewöhnlich 6-10 Minuten. Zur Auflösung einiger in Wasser schwer löslicher Polypeptide verwendeten wir einige Tropfen Natronlauge. Im übrigen wurden die Substanzen in $10 \mathrm{ccm}$ Wasser gelöst.

Die folgende Tabelle gibt einen Überblick über die erhaltenen Resultate:

1) Donald D. van Slyke, Eine Methode zur quantitativen Bestimmung der aliphatischen Aminogruppen; einige Anwendungen derselben in der Chemie der Proteine, des Harns und der Enzyme. Berichte der Deutsch. chem. Gesellsch., Jg. 43, S. 3170, 1910.

2) Emil Abderhalden, Partielle Hydrolyse einiger Proteine, Diese Zeitschrift, Bd. 58, S. 373, 1908.

s) Ein Gemisch von Isomeren ist natürlich nicht ausgeschlossen.

4) Vgl. über das erste Tripeptid: Emil Abderhalden, Weiterer Beitrag zur Kenntnis der bei der partiellen Hydrolyse von Proteinen entstehenden Spaltprodukte, Diese Zeitschrift, Bd. 72, S. 1, 1911. 
Tabelle 1.

\begin{tabular}{|c|c|c|c|c|c|c|c|}
\hline & \multirow[b]{2}{*}{$\begin{array}{c}\text { Sub- } \\
\text { stanz } \\
\text { in } g\end{array}$} & \multirow[b]{2}{*}{$\begin{array}{l}\mathrm{N} \text {-Gas } \\
\text { in } \mathrm{ccm} \\
\end{array}$} & \multirow[b]{2}{*}{$\begin{array}{l}\text { Tem- } \\
\text { peratur } \\
\text { in }{ }^{\circ} \mathrm{C} .\end{array}$} & \multirow[b]{2}{*}{$\begin{array}{l}\text { Druck } \\
\text { in } \mathrm{mm} \\
\end{array}$} & \multicolumn{2}{|c|}{ Amino-N } & \multirow{2}{*}{$\begin{array}{l}\text { Gesamt- } \\
\mathrm{N} \\
\text { (berech- } \\
\text { netin \%) }\end{array}$} \\
\hline & & & & & \begin{tabular}{|c|} 
ge- \\
funden \\
in $\%$ \\
\end{tabular} & $\begin{array}{c}\text { be- } \\
\text { rechnet } \\
\text { in } \%\end{array}$ & \\
\hline \multirow{2}{*}{$\begin{array}{l}\text { I. Aminosäuren. } \\
\text { Glukosaminsäure } \mathrm{C}_{6} \mathrm{H}_{11} \mathrm{O}_{6} \mathrm{~N} \\
\text { II. Dipeptide. }\end{array}$} & 0,0961 & 12,35 & 29 & 757 & 7,10 & 7,26 & 7,26 \\
\hline & & & & & & & \\
\hline Glycyl-asparagin $\mathrm{C}_{6} \mathrm{H}_{11} \mathrm{O}_{4} \mathrm{~N}_{3}$ & 0,0951 & 16,05 & 29 & 757 & $\mathbf{9 , 1 2}$ & 7,41 & 22,23 \\
\hline $\begin{array}{l}\text { Glycyl-l-phenyl- } \\
\text { alanin }\end{array}$ & 0,0778 & 10,90 & 25,5 & 759 & 7,74 & 6,34 & 12,68 \\
\hline$\underset{\text { phan }}{\text { Glyl-trypto- }} \quad \mathrm{C}_{18} \mathrm{H}_{15} \mathrm{O}_{3} \mathrm{~N}_{3}$ & 0,0297 & 3,25 & 26 & 752 & 5,96 & $\mathbf{5 , 3 6}$ & 16,07 \\
\hline \multirow{2}{*}{$\begin{array}{l}\text { Alanyl-glycin } \mathrm{C}_{5} \mathrm{H}_{10} \mathrm{O}_{3} \mathrm{~N}_{2} \\
\text { d-Alanyl-glycin aus } \\
\text { Seide }\end{array}$} & 0,0994 & 17,90 & 28 & 760 & $\mathbf{9 , 8 3}$ & 9,60 & 19,20 \\
\hline & 0,1021 & 17,80 & 27 & 757 & $\mathbf{9 , 4 6}$ & 9,60 & 19,20 \\
\hline Alanyl-alanin & 0,1060 & 15,90 & 25,5 & 759 & 8,49 & 8,75 & 17,50 \\
\hline Alanyl-leucin $\quad \mathrm{C}_{9} \mathrm{H}_{18} \mathrm{O}_{9} \mathrm{~N}_{2}$ & 0,1004 & 13,50 & 28 & 758 & 7,32 & 6,94 & 13,88 \\
\hline $\begin{array}{l}\text { Aminobutyryl-amino- } \\
\text { buttersäure } \mathrm{C}_{8} \mathrm{H}_{18} \mathrm{O}_{8} \mathrm{~N}_{2}\end{array}$ & 0,1070 & 14,10 & $25, \check{5}$ & 759 & $\mathbf{7 , 2 3}$ & $\mathbf{7 , 4 5}$ & 14,90 \\
\hline Valyl-glycin $\quad \mathrm{C}_{7} \mathrm{H}_{14} \mathrm{O}_{3} \mathrm{~N}_{2}$ & 0,0984 & 14,20 & 28 & 759 & 7,76 & 8,05 & 16,09 \\
\hline Valyl-alanin & 0,0539 & 7,30 & 28 & 759 & 7,38 & 7,45 & 14,90 \\
\hline Leucyl-isoserin $\mathrm{C}_{9} \mathrm{H}_{18} \mathrm{O}_{4} \mathrm{~N}_{2}$ & 0,0961 & 11,90 & 28 & 760 & 6,76 & 6,43 & 12,86 \\
\hline $\begin{array}{l}\text { Leucyl-tyrosin- } \\
\text { anhydrid }\end{array} \mathrm{C}_{15} \mathrm{H}_{22} \mathrm{O}_{4} \mathrm{~N}_{8}$ & 0,0597 & 0,00 & - & - & 0,00 & 0.00 & 9,52 \\
\hline$\underset{\text { glycin }}{\text { Phenylalanyl- }} \quad \mathrm{C}_{11} \mathrm{H}_{14} \mathrm{O}_{8} \mathrm{~N}_{2}$ & 0,1004 & 12,20 & 30 & 760 & 6,64 & 6,34 & 12,68 \\
\hline III. Tripeptid. & & & & & & & \\
\hline Diglycyl-glycin $\quad \mathrm{C}_{6} \mathrm{H}_{11} \mathrm{O}_{4} \mathrm{~N}_{3}$ & 0,0968 & 17,10 & 29 & 7508 & $\mathbf{9 , 5 6}$ & $\mathbf{7 , 4 1}$ & 22,24 \\
\hline $\begin{array}{l}\text { Alanyl-glycyl- } \\
\text { glycin }\end{array}$ & 0,1061 & 12,60 & 26,5 & 759 & $\mathbf{6 , 5 3}$ & 6,90 & 20,71 \\
\hline$\underset{\text { glycin }}{\text { Alanyl-leucyl- }} \quad \mathrm{C}_{11} \mathrm{H}_{21} \mathrm{O}_{4} \mathrm{~N}_{8}$ & 0,0947 & 9,90 & 29 & 758 & 5,66 & $\mathbf{5 , 4 1}$ & 16,21 \\
\hline$\underset{\text { glycin }}{\text { Leucyl-glycyl- }} \quad \mathrm{C}_{10} \mathrm{H}_{19} \mathrm{O}_{4} \mathrm{~N}_{3}$ & 0,0992 & 10,40 & 28 & 755 & $\mathbf{5 , 6 8}$ & $\mathbf{5 , 7 1}$ & 17,14 \\
\hline$\underset{\text { glycin }}{\text { l-Leucyl-glycyl- }} \mathrm{C}_{10} \mathrm{H}_{19} \mathrm{O}_{4} \mathrm{~N}_{8}$ & 0,0485 & 5,20 & 26 & 752 & $\mathbf{5}, \mathbf{8 5}$ & $\mathbf{5 , 7 1}$ & 17,14 \\
\hline $\begin{array}{l}\text { 1-Leucyl-1-tryptophyl- } \\
\text { d-glutaminsäure }{ }^{1} \\
\text { aus Edestin } \\
\mathrm{C}_{22} \mathrm{H}_{30} \mathrm{O}_{6} \mathrm{~N}_{4}\end{array}$ & 0,0338 & 2,03 & 27 & 757 & $\mathbf{3}, 25$ & 3,14 & 12,56 \\
\hline
\end{tabular}

1) Struktur fraglich! 
Tabelle 1.

Fortsetzung.

\begin{tabular}{|c|c|c|c|c|c|c|c|}
\hline & \multirow[b]{2}{*}{$\begin{array}{l}\text { Sub- } \\
\text { stanz } \\
\text { in } g\end{array}$} & \multirow[b]{2}{*}{$\begin{array}{l}\mathrm{N}-\mathrm{Gas} \\
\text { in } \mathrm{ccm}\end{array}$} & \multirow[b]{2}{*}{$\begin{array}{l}\text { Tem- } \\
\text { peratur } \\
\text { in }{ }^{0} \mathrm{C} .\end{array}$} & \multirow[b]{2}{*}{$\begin{array}{l}\text { Druck } \\
\text { in } \mathrm{mm}\end{array}$} & \multicolumn{2}{|c|}{ Amino-N } & \multirow{2}{*}{\begin{tabular}{|c|} 
Gesamt- \\
N \\
(berech- \\
net in $\%)$
\end{tabular}} \\
\hline & & & & & $\begin{array}{l}\text { ge- } \\
\text { funden } \\
\text { in } \%\end{array}$ & $\begin{array}{c}\text { be- } \\
\text { rechnet } \\
\text { in } \%\end{array}$ & \\
\hline IV. Tetrapeptide. & & & & & & & \\
\hline Triglycyl-glycin $\mathrm{C}_{8} \mathrm{H}_{14} \mathrm{O}_{5} \mathrm{~N}_{4}$ & 0,1037 & 12,90 & 26 & 752 & 6,79 & 5,69 & 22,77 \\
\hline$\underset{\text { glycin }}{\text { Leucyl-diglycyl- }} \mathrm{C}_{12} \mathrm{H}_{22} \mathrm{O}_{5} \mathrm{~N}_{4}$ & 0,0482 & 4,20 & 28 & 755 & $\mathbf{4 , 7 4}$ & 4,64 & 18,56 \\
\hline $\begin{array}{c}\text { V. Hochmolekulare } \\
\text { Polypeptide. }\end{array}$ & & & & & & & \\
\hline $\begin{array}{l}\text { l-Leucyl-octa- } \\
\text { glycyl-glycin }\end{array} \mathrm{C}_{\mathbf{2 4}} \mathrm{H}_{40} \mathrm{O}_{\mathbf{1 1}} \mathrm{N}_{10}$ & 0,0700 & 2,50 & 26 & 757 & 1,96 & 2,17 & 21,75 \\
\hline $\begin{array}{l}\text { l-Leucyl-triglycyl- } \\
\text { 1-leucyl-octaglycyl- } \\
\text { glycin } \mathrm{C}_{\mathbf{3 6}} \mathrm{H}_{60} \mathrm{O}_{15} \mathrm{~N}_{14}\end{array}$ & 0,0777 & 2,10 & 27 & 757 & $1, \mathbf{4 7}$ & $1, \mathbf{5 1}$ & 21,13 \\
\hline
\end{tabular}

Nach den bisherigen Erfahrungen erhält man bei den Polypeptiden, an deren Aufbau Glykokoll, das die Aminogruppe trägt, beteiligt ist, aus den gefundenen Werten annähernd die richtigen, wenn man diese mit dem Faktor 0,8 multipliziert. Vgl. die folgende Tabelle.

Tabelle 2.

Korrektur für die Glycyl-Polypeptide.

\begin{tabular}{|c|c|c|c|}
\hline \multirow[b]{2}{*}{. } & \multicolumn{3}{|c|}{ Aminosticksoff } \\
\hline & gefunden & $\begin{array}{l}\text { gefundener } \\
\text { Wert } \times 0,8\end{array}$ & berechnet \\
\hline ............ & 13,7 & 10,96 & 10,60 \\
\hline Glycyl-phenylalanin . . . . . . . . & 7,74 & 6,19 & 6,34 \\
\hline Glycyl-asparagin . . . . . . . . . . & 9,12 & 7,30 & 7,41 \\
\hline Glycyl-tryptophan (nur 0,0297 g zur Analyse) & 5,96 & 4,77 & 5,36 \\
\hline Diglycyl-glycin. . . . . . . . . . & 9,56 & 7,65 & 7,41 \\
\hline Triglycyl-glycin . . . . . . . . . . & 6,79 & 5,43 & 5,69 \\
\hline
\end{tabular}

